\title{
Triangle congruence and the Moulton plane
}

\author{
Robert C. Powers and Prasanna K. Sahoo \\ Robert C. Powers received his Ph.D. in mathematics from the University of Mas- \\ sachusetts in 1988. Since 1988 he is professor of mathematics at the University of \\ Louisville in Louisville, Kentucky. He has published papers in discrete mathematics, \\ algebra and college geometry. Outside of mathematics, he enjoys spending time with \\ his wife and two daughters. \\ Prasanna K. Sahoo received his Ph.D. in applied mathematics from the University \\ of Waterloo, Canada in 1986. Since 1987 he is professor of mathematics at the Uni- \\ versity of Louisville in Louisville, Kentucky. He is a co-author of two books related \\ to functional equations and he has published papers in functional equations and their \\ applications to geometry, analysis, statistics, and information theory. Occasionally he \\ publishes papers in image processing and nonlinear pricing theory. Recently he has \\ co-authored a paper in The Journal of Business titled Buffet Pricing which received \\ the best paper award in 1997 at the annual meeting of the Kentucky Economic As- \\ sociation. Outside of mathematics, he enjoys spending time with his wife and son.
}

A protractor geometry is a model $\{\mathscr{P}, \mathscr{L}, d, m\}$ of plane geometry such that $\{\mathscr{P}, \mathscr{L}, d\}$ is a metric geometry satisfying Pasch's axiom and $m$ is an angle measure [2]. In this context, $\mathscr{P}$ is the set of points, $\mathscr{L}$ is the set of lines, and $d$ is a distance function on $\mathscr{P}$. Pasch's axiom states that if a line intersects one side of a triangle then it must intersect at least one of the two remaining sides of the triangle. The segments $\overline{A B}$ and $\overline{D E}$ are congruent, denoted by $\overline{A B} \cong \overline{D E}$, if $d(A, B)=d(D, E)$ and angles $\angle A B C$ and $\angle D E F$

Laufen die Verbindungsgeraden entsprechender Eckpunkte zweier Dreiecke durch einen Punkt, so liegen nach dem Satz von Desargues die drei Schnittpunkte entsprechender Dreiecksseiten oder derer Verlängerungen auf einer Geraden. Im Gegensatz dazu begründete F.R. Moulton im Jahr 1902 ein einfaches Modell einer ebenen Geometrie, für das der Satz von Desargues verletzt ist. In diesem Modell, der sogenannten MoultonEbene, lässt sich nun auch eine Längen- und Winkelmessung erklären. Damit kann nach der Übertragbarkeit bzw. Verallgemeinerung der bekannten Dreieckskongruenzsätze der Euklidischen in die Moulton-Geometrie gefragt werden. Im nachfolgenden Beitrag wird in diesem Zusammenhang gezeigt, dass in der Moulton-Geometrie Kongruenzsätze, welche sowohl Seiten als auch Winkel von Dreiecken beinhalten, nicht gelten können. 
are congruent, denoted by $\angle A B C \cong \angle D E F$, if $m(\angle A B C)=m(\angle D E F)$. If $\triangle A B C$ and $\triangle D E F$ are two triangles in a protractor geometry $\{\mathscr{P}, \mathscr{L}, d, m\}$ then $\triangle A B C$ and $\triangle D E F$ are said to be congruent, denoted by $\triangle A B C \cong \triangle D E F$, if $\overline{A B} \cong \overline{D E}, \overline{B C} \cong \overline{E F}$, $\overline{A C} \cong \overline{D F}, \angle A \cong \angle D, \angle B \cong \angle E$, and $\angle C \cong \angle F$. What can be said about triangle congruence in the context of protractor geometry?

In [1], for instance, J. Boone investigates a protractor geometry where the four triangle congruence conditions (SAS), (ASA), (SAA), and (SSS) fail. Boone refers to this model as the Moise plane since he found it in Moise's book [3]. The Moise plane is the standard Euclidean plane except that the distance between two points on the $x$-axis is twice their Euclidean distance. It is possible to find two noncongruent triangles in the Moise plane such that three angles and two sides of the first triangle are congruent to three angles and two sides of the second triangle. So the Moise plane does not satisfy the triangle congruence condition (AAASS). In general, a protractor geometry $\{\mathscr{P}, \mathscr{L}, d, m\}$ satisfies Angle-Angle-Angle-Side-Side (AAASS) if, for any two triangles $\triangle A B C$ and $\triangle D E F, \angle A \cong \angle D, \angle B \cong \angle E, \angle C \cong \angle F, \overline{A C} \cong \overline{D F}$, and $\overline{B C} \cong \overline{E F}$ imply that $\triangle A B C \cong \triangle D E F$. On the other hand, it is not hard to see that in the Moise plane if three sides and two angles of one triangle are congruent to three sides and two angles of another triangle then the remaining angles are congruent and so the two triangles are congruent. That is, the Moise plane satisfies the triangle congruence condition (SSSAA). In general, a protractor geometry $\{\mathscr{P}, \mathscr{L}, d, m\}$ satisfies Side-SideSide-Angle-Angle (SSSAA) if, for any two triangles $\triangle A B C$ and $\triangle D E F, \overline{A B} \cong \overline{D E}$, $\overline{B C} \cong \overline{E F}, \overline{A C} \cong \overline{D F}, \angle A \cong \angle D$, and $\angle B \cong \angle E$ imply that $\triangle A B C \cong \triangle D E F$. Is (SSSAA) a property common to all protractor geometries? The answer is no. In fact, we will show that the Moulton plane is an example of a protractor geometry that does not satisfy any triangle congruence criteria involving sides and angles.

The Moulton plane [4] is best known as an example of an affine (projective) plane where Desargues' theorem does not hold. The underlying set of points is $\mathbb{R}^{2}$. A line in the Moulton plane is either a Euclidean line with nonpositive slope, a vertical Euclidean line, or is a set of the form

$$
\left\{(x, y) \in \mathbb{R}^{2} \mid y=m x+b \text { if } x \leq 0, \quad y=\frac{1}{2} m x+b \text { if } x>0, \text { and } m>0\right\} .
$$

The idea is that a Moulton line with positive slope gets bent as it passes across the $y$-axis (see line $P_{1} Q_{1}$ in Figure 1) and a Moulton line with negative slope is the same as the Euclidean line (see line $P_{2} Q_{2}$ in Figure 1). It is not easy to geometrically construct the Moulton line $P_{1} Q_{1}$, and in fact, a computational approach is more convenient. The set of Moulton lines is denoted by $\mathscr{L}_{M}$. It should be noted that Moulton lines are the geodesics in this geometry.

If $d_{E}$ is the usual Euclidean distance function, then the Moulton distance between the points $P=\left(x_{1}, y_{1}\right)$ and $Q=\left(x_{2}, y_{2}\right)$ in $\mathbb{R}^{2}$ is given by

$$
d_{M}(P, Q)= \begin{cases}d_{E}(P,(0, b))+d_{E}((0, b), Q) & \text { if } P, Q, \text { and }(0, b) \text { are } \\ & \text { collinear and } x_{1} x_{2}<0 ; \\ d_{E}(P, Q) & \text { otherwise. }\end{cases}
$$




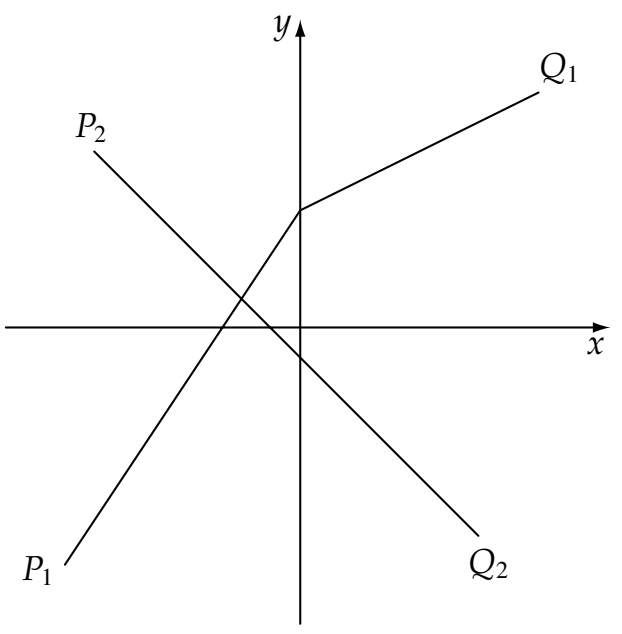

Fig. 1 Moulton lines

Angle measure for the Moulton plane is in terms of Euclidean angle measure $m_{E}$. If the point $B$ is not on the $y$-axis, then the Moulton measure of the angle $\angle A B C$ is given by $m_{M}(\angle A B C)=m_{E}\left(\angle A^{\prime} B C^{\prime}\right)$ where $\overrightarrow{B A}=\overrightarrow{B A^{\prime}}, \overrightarrow{B C}=\overrightarrow{B C^{\prime}}$, and $A^{\prime}, B$, and $C^{\prime}$ are on the same side of the $y$-axis (see Figure 2). If $B=(0, b), A=\left(x_{1}, y_{1}\right)$, and $C=\left(x_{2}, y_{2}\right)$, then the Moulton measure of the angle $\angle A B C$ is given by $m_{M}(\angle A B C)=m_{E}\left(\angle A^{\prime} B C^{\prime}\right)$ where

$$
A^{\prime}= \begin{cases}\left(x_{1}, 2 y_{1}-b\right) & \text { if } x_{1}>0 \text { and } y_{1}>b \\ \left(x_{1}, y_{1}\right) & \text { otherwise }\end{cases}
$$

and

$$
C^{\prime}= \begin{cases}\left(x_{2}, 2 y_{2}-b\right) & \text { if } x_{2}>0 \text { and } y_{2}>b \\ \left(x_{2}, y_{2}\right) & \text { otherwise. }\end{cases}
$$

For example, using the top triangle in Figure 3 we have

$$
m_{M}(<A C B)=m_{E}\left(<A^{\prime} C B^{\prime}\right)=\tan ^{-1}(\sqrt{2})
$$

where $A^{\prime}=\left(\frac{\sqrt{2}+2}{3}, \frac{2 \sqrt{2}+2}{3}\right)$ and $B^{\prime}=B$.

It is a tedious exercise to show that $\left\{\mathbb{R}^{2}, \mathscr{L}_{M}, d_{M}, m_{M}\right\}$ is a protractor geometry (see [2]). Consequently, the Moulton plane satisfies the axioms and properties of a protractor geometry. So Pasch's axiom holds in the Moulton plane. The standard axioms that define incidence geometry are also true in the Moulton plane. In particular, there exists a unique line through any two distinct points. Hilbert's axioms on betweenness hold in the Moulton plane. For example, for any three distinct collinear points exactly one of these point lies between the other two. The Moulton plane satisfies the Euclidean parallel property. Consequently, for any line $\ell$ and for any point $P$ not on $\ell$ there exists a unique line $m$ incident with $P$ parallel to $\ell$. This last property is not true for all protractor geometries. 


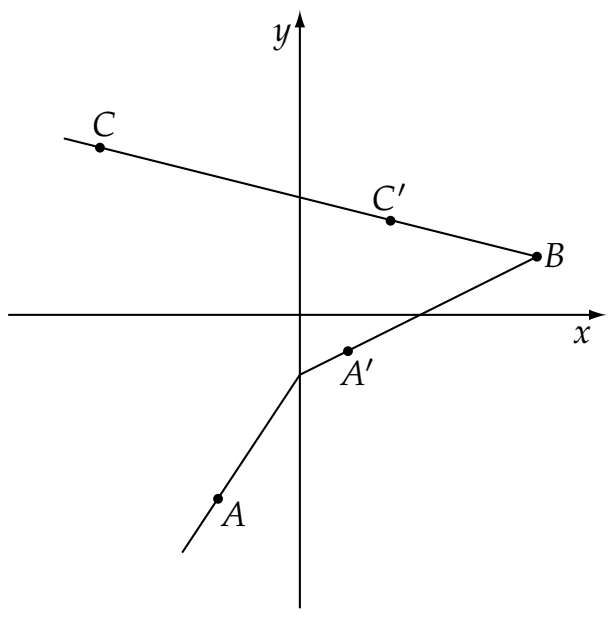

Fig. 2 Moulton angle measure

This model has some bizarre properties as well. For example, the sum of the angle measures of a Moulton triangle could be greater than $180^{\circ}$. This is the case for the two triangles given in Figure 3. On the other hand, there exist triangles whose angle sums are less than $180^{\circ}$. We invite the reader to find such a triangle. Another strange property is the failure of the triangle inequality. To see this, note that the points $(-1,-1),(0,0)$, and $(1,1)$ are not collinear in the Moulton plane and that

$$
\begin{aligned}
d_{M}((-1,-1),(0,0)) & +d_{M}((0,0),(1,1)) \\
& =d_{E}((-1,-1),(0,0))+d_{E}((0,0),(1,1)) \\
& =d_{E}((-1,-1),(1,1)) \\
& <d_{E}\left((-1,-1),\left(0, \frac{1}{3}\right)\right)+d_{E}\left(\left(0, \frac{1}{3}\right),(1,1)\right) \\
& =d_{M}((-1,-1),(1,1)) .
\end{aligned}
$$

A final example is the failure of the Pythagorean theorem. To illustrate this point observe that $m_{M}\left(\angle(-1,1)(0,0)\left(1, \frac{1}{2}\right)\right)=90^{\circ}$ and that

$$
\begin{aligned}
\frac{13}{4} & =d_{M}((-1,1),(0,0))^{2}+d_{M}\left((0,0),\left(1, \frac{1}{2}\right)\right)^{2} \\
& <d_{M}\left((-1,1),\left(1, \frac{1}{2}\right)\right)^{2}=\frac{17}{4}
\end{aligned}
$$

We now give two examples that will show that the Moulton plane is a protractor geometry not satisfying any triangle congruence criteria involving sides and angles. The first example shows that the Moulton plane does not satisfy (SSSAA) whereas the second, and the more challenging example, shows that (AAASS) fails. 
Since

$$
m_{M}(\angle(1,1)(0,0)(1,0))=m_{E}(\angle(1,2)(0,0)(1,0))=\tan ^{-1}(2)
$$

and

$$
m_{M}(\angle(1,1)(2,0)(1,0))=45
$$

the triangles $\triangle(0,0)(1,0)(1,1)$ and $\triangle(1,1)(2,0)(1,0)$ are not congruent in the Moulton plane. However, the remaining corresponding angles and sides are congruent.

The triangles $\triangle A B C$ and $\triangle A^{\prime} B^{\prime} C^{\prime}$ whose vertices are

$$
A=\left(\frac{\sqrt{2}+2}{3}, \frac{\sqrt{2}+1}{3}\right), \quad B=(1,0), \quad C=(0,0)
$$

and

$$
A^{\prime}=\left(\frac{2 \sqrt{2}}{3}, \frac{2}{3}\right), \quad B^{\prime}=(0,0), \quad C^{\prime}=\left(\frac{1}{\sqrt{2}},-\frac{1}{\sqrt{2}}\right),
$$

respectively, are illustrated in Figure 3. These two triangles are not congruent in the Moulton plane since $d_{M}(A, B)=\frac{\sqrt{6}}{3}$ and $d_{M}\left(A^{\prime}, B^{\prime}\right)=\frac{\sqrt{12}}{3}$. So $\overline{A B} \approx \overline{A^{\prime} B^{\prime}}$. On the other hand, $\overline{B C} \cong \overline{B^{\prime} C^{\prime}}$ and $\overline{A C} \cong \overline{A^{\prime} C^{\prime}}$ since $d_{M}(B, C)=d_{M}\left(B^{\prime}, C^{\prime}\right)=1$ and

$$
d_{M}(A, C)=d_{M}\left(A^{\prime}, C^{\prime}\right)=\frac{\sqrt{9+6 \sqrt{2}}}{3}=\frac{\sqrt{6}+\sqrt{3}}{3} .
$$

We now show that corresponding angles are congruent. We already noted that $m_{M}(\angle A C B)=\tan ^{-1}(\sqrt{2})$. Since $y=\frac{1}{\sqrt{2}} x$ is the unique Euclidean line through the points $A$ and $C$ it follows that $m_{E}(\angle A C B)=\tan ^{-1}\left(\frac{1}{\sqrt{2}}\right)$. By the Law of Sines,

$$
\frac{\sin (\angle C A B)}{1}=\frac{\sin \left(\tan ^{-1}\left(\frac{1}{\sqrt{2}}\right)\right)}{\frac{\sqrt{6}}{3}}=\frac{\frac{1}{\sqrt{3}}}{\frac{\sqrt{6}}{3}}=\frac{1}{\sqrt{2}} .
$$

So $m_{M}(\angle C A B)=m_{E}(\angle C A B)=45^{\circ}$. Since $\tan ^{-1}(\sqrt{2})+\tan ^{-1}\left(\frac{1}{\sqrt{2}}\right)=90^{\circ}$ it follows that

$$
\begin{aligned}
m_{M}(\angle A B C) & =m_{E}(\angle A B C) \\
& =180^{\circ}-m_{E}(\angle C A B)-m_{E}(\angle A C B) \\
& =180^{\circ}-45^{\circ}-\tan ^{-1}\left(\frac{1}{\sqrt{2}}\right) \\
& =\tan ^{-1}(\sqrt{2})+45^{\circ} .
\end{aligned}
$$

The Moulton measures of the three angles in $\triangle A B C$ are listed in Figure 3.

Consider the second triangle in Figure 3 with $D$ being the point where the line $A^{\prime} C^{\prime}$ intersects the $x$-axis. Since $y=\frac{1}{\sqrt{2}} x$ is the unique Euclidean line through the points 

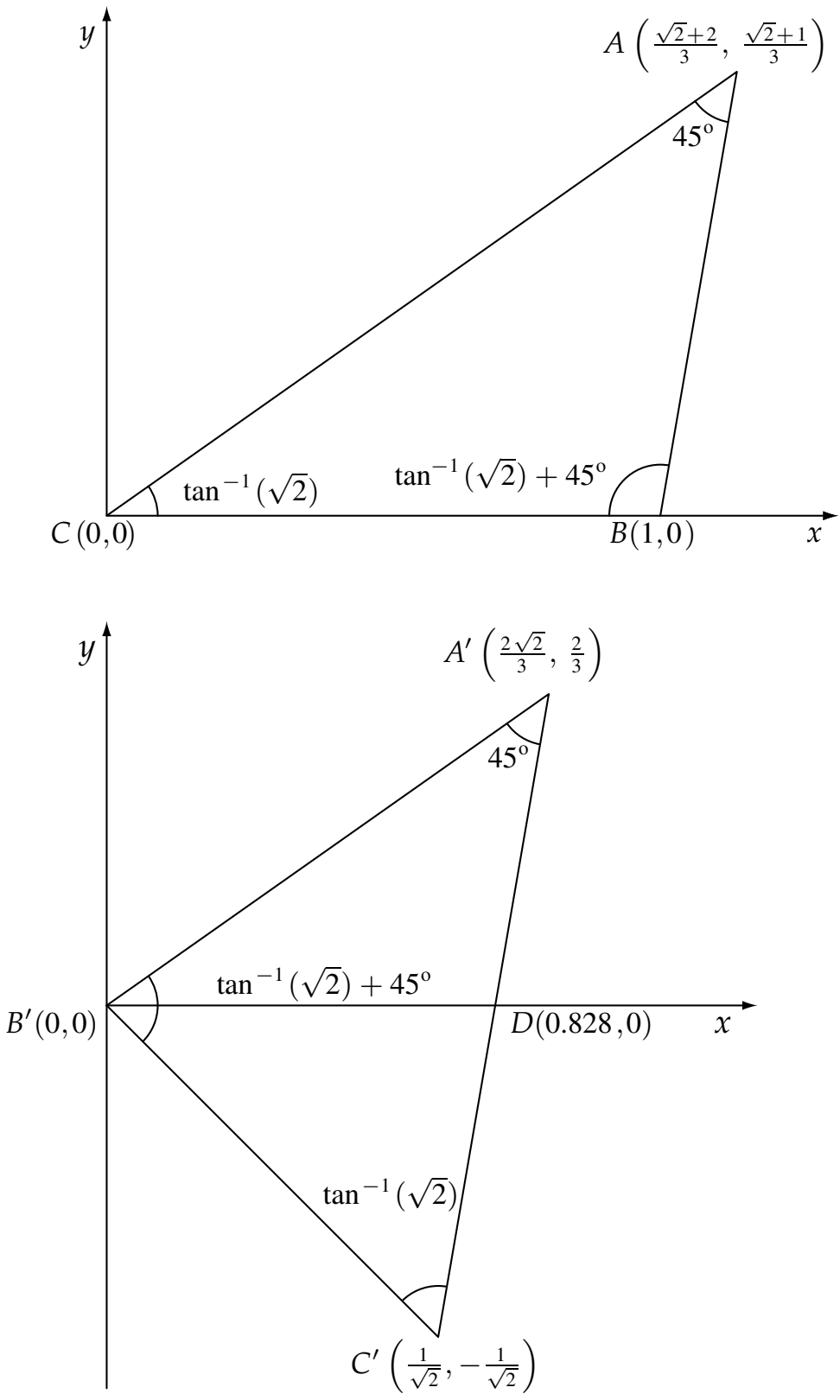

Fig. 3 (AAASS) fails in the Moulton plane 
$A^{\prime}$ and $B^{\prime}$ it follows (as above) that $m_{M}\left(\angle A^{\prime} B^{\prime} D\right)=\tan ^{-1}(\sqrt{2})$ and $m_{E}\left(\angle A^{\prime} B^{\prime} D\right)=$ $\tan ^{-1}\left(\frac{1}{\sqrt{2}}\right)$. So

$$
\begin{aligned}
m_{M}\left(\angle A^{\prime} B^{\prime} C^{\prime}\right) & =m_{M}\left(\angle A^{\prime} B^{\prime} D\right)+m_{M}\left(\angle D B^{\prime} C^{\prime}\right) \\
& =m_{E}\left(\angle A^{\prime} B^{\prime} D\right)+m_{E}\left(\angle D B^{\prime} C^{\prime}\right) \\
& =\tan ^{-1}(\sqrt{2})+45^{\circ} .
\end{aligned}
$$

A similar argument shows that $m_{E}\left(\angle A^{\prime} B^{\prime} C^{\prime}\right)=\tan ^{-1}\left(\frac{1}{\sqrt{2}}\right)+45^{\circ}$. By the Law of Sines,

$$
\frac{\sin \left(\angle C^{\prime} A^{\prime} B^{\prime}\right)}{1}=\frac{\sin \left(\tan ^{-1}\left(\frac{1}{\sqrt{2}}\right)+45^{\circ}\right)}{\frac{\sqrt{6}+\sqrt{3}}{3}} \text {. }
$$

Since

$$
\begin{aligned}
\sin \left(\tan ^{-1}\left(\frac{1}{\sqrt{2}}\right)+45^{\circ}\right) & =\sin \left(\tan ^{-1}\left(\frac{1}{\sqrt{2}}\right)\right) \cos \left(45^{\circ}\right)+\cos \left(\tan ^{-1}\left(\frac{1}{\sqrt{2}}\right)\right) \sin \left(45^{\circ}\right) \\
& =\frac{1}{\sqrt{3}} \frac{1}{\sqrt{2}}+\frac{\sqrt{2}}{\sqrt{3}} \frac{1}{\sqrt{2}},
\end{aligned}
$$

it follows that $\sin \left(\angle C^{\prime} A^{\prime} B^{\prime}\right)=\frac{\sqrt{2}}{2}$. So

$$
m_{M}\left(\angle C^{\prime} A^{\prime} B^{\prime}\right)=m_{E}\left(\angle C^{\prime} A^{\prime} B^{\prime}\right)=45^{\circ} .
$$

The last angle measure is given by

$$
\begin{aligned}
m_{M}\left(\angle A^{\prime} C^{\prime} B^{\prime}\right) & =m_{E}\left(\angle A^{\prime} C^{\prime} B^{\prime}\right) \\
& =180^{\circ}-m_{E}\left(\angle A^{\prime} B^{\prime} C^{\prime}\right)-m_{E}\left(\angle C^{\prime} A^{\prime} B^{\prime}\right) \\
& =180^{\circ}-\left(\tan ^{-1}\left(\frac{1}{\sqrt{2}}\right)+45^{\circ}\right)-45^{\circ} \\
& =\tan ^{-1}(\sqrt{2}) .
\end{aligned}
$$

Thus $\angle A \cong \angle A^{\prime}, \angle B \cong \angle B^{\prime}$, and $\angle C \cong \angle C^{\prime}$ and we are done.

Acknowledgment. The authors gratefully acknowledge several helpful comments of the referee that improved the presentation of this paper.

\section{References}

[1] Boone, J.R.: The Moise Plane. College Math. J., Vol. 27, No. 3, 1996.

[2] Millman, R.S. and Parker, G.D.: Geometry: A Metric Approach with Models, 2nd ed., Springer-Verlag, New York, 1991.

[3] Moise, E.E.: Elementary Geometry from an Advanced Standpoint, 3rd ed., Addison-Wesley, Reading, MA, 1990.

[4] Moulton, F.R.: A simple non-desarguesian plane geometry, Transactions of AMS 3 (1902), 192-195.

Robert C. Powers

Department of Mathematics

University of Louisville

Louisville, Kentucky 40292, USA

e-mail:

rcpowe01@athena. louisville.edu
Prasanna K. Sahoo

Department of Mathematics

University of Louisville

Louisville, Kentucky 40292, USA

e-mail:

sahoo@louisville.edu 